\title{
SUPERNOVAE AND NEUTRON STARS
}

\author{
STIRLING A. COLGATE \\ New Mexico Institute of Mining and Technology, Socorro, N.M. 87801, U.S.A.
}

\begin{abstract}
The formation of a neutron star and the event observed as a supernova are presumed by most astrophysicists to be simultaneous. The theoretical difficulty of understanding this is compounded by a hierarchy of conflicting phenomena dominated by the possibility of effective neutrino transport of the heat of the neutron star formation to the outer envelope. A thermonuclear detonation of a carbon core may take place at just the right density such that both neutron star formation and neutrino assisted envelope ejection can occur together.
\end{abstract}

\section{Supernova and Neutron Star: The Same Event?}

This paper reviews one particular problem of supernova theory; namely, how can the birth of a neutron star and a supernova be one and the same event and yet how can they not be?

The theories of supernovae are faced with a major dichotomy: on the one hand, we see a neutron star remnant at the site of one supernova, the Crab, and infer that many other cases are similar - yet on the other hand, serious doubt has been cast on the numerical theoretical calculations that might explain such events.

The problem centers on the requirement of simultaneously ejecting something like a solar mass at the same time that a similar mass collapses to a neutron star. First of all, what is the justification for both 'a solar mass' and 'near simultaneity'? The collapse to a neutron star requires that sufficient mass be assembled at a low enough temperature such that the available specific gravitational energy is greater than the specific energy required to convert a proton-electron pair into a neutron, when that proton is at the nuclear binding corresponding to that in helium. At the stellar densities where collapse to a neutron star is imminent, there is little question that stellar nucleosynthesis has proceeded to the point of at least helium - and more likely carbon-oxygen and even to the end point - iron. Ninety percent of the nuclear binding occurs in helium and so whatever additional synthesis has occurred will not significantly change the approximate result. The minimum energy required to convert $\mathrm{He}$ to free neutrons is about $10^{19} \mathrm{erg} \mathrm{g}^{-1}$. This must be equated to $M G / r$, the gravitational potential. If we want to minimize $M$, we must minimize the radius. The minimum radius in turn is set by the condition that we do not want the degenerate electron pressure to require more work in collapse than the conversion of protons to neutrons or put another way, protons will be converted to neutrons whenever the electron Fermi level becomes greater than the nuclear energy difference. This occurs at $\varrho \simeq 2 \times 10^{11} \mathrm{~g} \mathrm{~cm}^{-3}\left(E_{\mathrm{Fermi}} \simeq 10 \mathrm{MeV}\right)$ which is therefore the density of incipient collapse. Using this density results in $r \simeq 10^{7} \mathrm{~cm}$ and $M \simeq M_{\odot}$. If the temperature is raised (compared with $E_{\mathrm{F}} \simeq 10 \mathrm{MeV}$ ) the minimum mass will be increased. The result is comfortably assuring in that we know that it requires a mass greater than the 
Chandrasekhar limit $1.13 M_{\odot}$ to overcome electron degeneracy pressure and reach this density in the first place, which, anyhow, is a similar way of looking at the condition for collapse.

The estimate of the ejection of a solar mass is an observational estimate derived from the light curves. One observes a peak luminosity and temperature; granted that these observations have some considerable error and even variation as to class of supernova, but for a typical type I supernova the peak luminosity may be $2 \times 10^{43} \mathrm{erg} \mathrm{s}^{-1}$ for a surface temperature $\simeq 10^{4} \mathrm{~K}$. The surface area required, $A(c / 4) a T^{4}=L$, becomes $2 \times 10^{31} \mathrm{~cm}^{2}$. The absolute minimum mass would be this area divided by the optical opacity. At the necessarily low density, this is Compton opacity, $10^{32} \mathrm{~g}$ for the surface layer. Models describing the mechanism of producing the optical radiation require some finite depth of the expanding (observed by Doppler shift) matter which at a minimum may be 10 mean free paths thick (in order that the diffusive release time of the radiation be as long as the observed width of one week) implying that at least $1 / 2 M_{\odot}$ is ejected.

Finally, we are presuming that these events occur nearly simultaneously. Extrapolation of the slowing down of the Crab pulsar leads to an origin within years of the supernova event. One could assume ejection took place (say 10 years?) before or after collapse; namely, that the events are only secondarily related, but the theoretical difficulties become even more staggering.

If ejection comes after collapse, then the matter must be lifted from the neutron star surface at the bottom of a gravitational well of greater than $100 \mathrm{MeV}$ per nucleon yet be observed 'dribbling' out into space at 1 to $2 \mathrm{MeV}$ per nucleon (maximum bulk expansion velocity $\simeq 2 \times 10^{9} \mathrm{~cm} \mathrm{~s}^{-1}$ ). No energy source has been proposed that could possibly achieve this. Ejection before collapse is more difficult to exclude. Conceivably, a thermonuclear detonation of a carbon-oxygen layer could take place at the surface of a more highly evolved, say, silicon core. The outer layer might be ejected and the smaller energy release in the core might be too small to disrupt the core and leave a large enough mass that later evolution would lead to collapse. The subtlety of conditions that would lead to thermonuclear detonation and partial disruption without simultaneously triggering collapse would seem hard to arrange - almost contrived and so unless there is overwhelming evidence to the contrary, most would agree that despite current difficulties, collapse and ejection must occur in the same dynamical event, but it is difficult to manage both at the current sophistication of theoretical analysis.

The original concept of achieving both events simultaneously depends upon utilizing the binding energy of the neutron star $(\simeq 100 \mathrm{MeV}$ per nucleon) to eject an outer, less strongly bound ( $1 \mathrm{MeV}$ per nucleon) mass fraction. We emphasize net binding energy, the difference between the internal and gravitational energies.

If comparable masses are ejected and collapsed, it would seem that the large energy difference would be more than adequate to achieve partial ejection. However, the difficulty arises in the inefficiency of energy transfer. If initially cold matter of relatively small binding energy were to collapse to a neutron star, the difference in binding 
energy would have to appear in internal degrees of freedom - oscillations and ultimately heat. Because of the propensity for the 'cold' fluid to form a shock wave during collapse, the formation process is relatively inelastic; the 'bounce' is weak, and essentially all the binding energy appears as heat. This heat, in turn, can be conducted to the outer layer by 'radiation' transport, heating the outer layers to the point where they blow off. The problem is that ordinary Planck radiation diffuses so slowly (the characteristic time is the Helmholtz contraction time of $10^{5} \mathrm{yr}$ ) that the outer layers would have long since collapsed in seconds onto the neutron star core and possibly exceeding the stable mass limit and forming a black hole. Fortunately, for the theoretician, at the high temperature of the just-formed neutron star of $\simeq 30 \mathrm{MeV}\left(3 \times 10^{11} \mathrm{~K}\right.$ or $100 \mathrm{MeV}$ per nucleon) radiation must include neutrinos as leptons in thermodynamic equilibrium and this equilibrium occurs in times of $10^{-8}$ to $10^{-6} \mathrm{~s}$ depending upon density, temperature and which kind of neutrino is involved, electron or muon. The thickness of the imploding matter onto the forming neutron star is variously one to ten mean free paths for the electron neutrinos and much less than one mean free path for the muon neutrinos. Therefore, only the electron neutrinos can transport heat to the outer layers and two conditions can prevent or greatly reduce this heat transport, and hence prevent sizeable mass ejection. If the imploding matter is thick enough (about 10 mean free paths) so that the neutrino radiation diffusion velocity $(\simeq c / 10)$ is less than the implosion velocity, then the diffusing heat will be continuously carried back to the neutron star and the outer layers will not be heated. Similarly, if the muon emission is great enough, it will allow the escape of the heat without interaction with the outer layers. Fortunately, one effect argues strongly in favor of some partial heat transport. As the temperature falls, so also does the neutrino electron scattering cross section, $\sigma_{v} \propto \mathrm{E}^{2}$, and with nuclear thresholds and degeneracy $\sigma_{v} \propto E^{4-5}$ so that decreasing temperature allows a more rapid (longer mean free path) diffusion of heat. In addition, a lower temperature favors electron neutrino emission as opposed to muon neutrino emission. The actual temperature and ratio of electron to muon neutrinos depend upon details of the hydrodynamics, but some of these details become crucial to the final outcome.

Initially, a simple transport of the neutrino heat from the standing shock in the imploding matter on the neutron star core appeared to give ample energy to eject the envelope at several $\mathrm{MeV}$ per nucleon. However, later calculations with more detailed neutrino transport negated this conclusion and only stars of mass greater than 4 to 6 solar masses ejected an envelope, and even so the residual core masses were greater than $2.5 M_{\odot}$. A hierarchy of effects could alter this unhappy state of affairs:

(i) The standing shock on the neutron core star preferentially emits the 'binding energy' in electron neutrinos in several meters equilibrium distance before muon neutrinos are emitted in several hundred meters distance.

(ii) In larger mass stars, the temperature immediately prior to collapse at $\varrho \sim 2 \times 10^{11} \mathrm{~g} \mathrm{~cm}^{-3}$ makes a sensitive difference to the hydrodynamics during the near free fall to the neutron star surface. This matter is cooled just before collapse by thermal decomposition to proton rich nuclei with subsequent electron capture, 
neutrino emission and hence cooling to a lower adiabat during collapse. The lower adiabat leads to a stronger standing shock, lower neutrino opacity in the collapsing matter and hence a larger heating of the external layers.

Finally, to confound the issue further, collapse calculations have been usually performed with relatively large and finite mass zoning so that not only is the detail of the various shocks neglected, i.e., electron vs. muon neutrino emission, but in the expanding phase after the envelope is heated and starts to expand some one inner mass zone developes a large ratio of inner and outer boundary radii. Further collapse onto the neutron star is halted due to this artifact independent of further neutrino cooling or what-have-you. An analytical approximation of the explosion phase indicates that for a very wide range of explosion conditions, wider than the most optimistic explosion advocates would claim, one-half the mass of matter initially ejected will fall back onto the neutron star. This is because in a spherical explosion, the inner regions perforce must expand relatively slowly. When the gravitational and thermal sink of a neutron star is introduced at the center, collapse of even the hottest matter back onto the neutron star is assured. Although this secondary collapse of matter, which might have experienced transient densities of $10^{9} \leqslant \varrho \leqslant 10^{12} \mathrm{~g} \mathrm{~cm}^{-3}$ is comforting to those who worry about the possibility of producing too much neutron rich matter for the Galaxy, nevertheless, the fact that only one-half the mass is really ejected compared to what we used to think, makes simultaneous ejection of a solar mass and collapse of less than $2 M_{\odot}$ even more difficult.

\section{Thermonuclear Detonations}

In recent years, as well as at the start of all theoretical considerations of supernova mechanisms, the detonation of an evolving carbon star has been an attractive way to make supernova, heavy elements, etc. However, for awhile it seemed that calculations indicated that despite the relatively modest overpressure $(\simeq 10 \%)$ created by the thermonuclear burning of carbon to completion (a mixture of all nuclear species up to about iron), still the star seemed to disrupt itself completely leaving no neutron star remnant! In order to conceivably form a neutron star, the matter had to reach several $\times 10^{10} \mathrm{~g} \mathrm{~cm}^{-3}$ before detonation so that beta decays could take place fast enough (due to the high electron Fermi level) so that collapse took place before explosion. Such a high density seemed impossible to reach without prior detonation of carbon at several $\times 10^{9} \mathrm{~g} \mathrm{~cm}^{-3}$. Again, a new theory has saved the day - one which predicts convective URCA shells with sufficiently steep temperature dependence as to overcome the carbon-carbon reaction sensitivity.

It now appears that a star can evolve stably through carbon burning up to the end point of iron and hence collapse with no nuclear energy available. A more attractive viewpoint is that collapse is initiated when there is still potentially explosive fuel, carbon-oxygen in the outer layers of the presupernova core, so that detonation and collapse to a neutron star occur nearly simultaneously. Eureka! But not quite. Long ago this was tried numerically where $5 M_{\odot}$ of equivalent carbon-carbon burning 
energy were dumped into the outer layers of a $10 M_{\odot}$ star at the time when the inner part started collapsing to a neutron star. It all fell in unless neutrino transport was added. In other words, the overpressure created by a carbon detonation is so small (because of the high specific heat of the many nuclear species and radiation) that the rarefaction created by an inner collapse completely dominates the explosion and turns the matter around to an implosion. There the matter currently rests - in neutron stars, black holes - wherever. Perhaps a subtle combination of detonation in the outer layers, retarded implosion and hence lower density in the matter imploding onto the neutron star will cause a strong enough standing shock such that the electron neutrino heat can escape fast enough to boost the carbon-carbon detonation in the outer layers sufficiently to eject part of these detonating outer layers - and form us.

\section{Acknowledgements}

I am deeply indebted to the many original and creative scientists that have conceived and written the original papers from which this discussion was drawn. Foremost among them are: William Fowler, Fred Hoyle, Geoffrey Burbidge, Margaret Burbidge, Al Cameron, Jim Truran, Dave Arnett, Bob Schwartz, B. Paczynski, G. Rakavy, G. Shaviv, Jim Fraley, Don Clayton, Dave Schramm, Dick White, Jim Wilson, M. LeBlanc, Carl Hansen, Craig Wheeler, Zalman Barkat, J.-R. Buchler, Steve Bruenn, Chester McKee, and others.

\section{DISCUSSION}

Van Horn: Since the problem of black holes has been raised I would like to ask the following question: situations must arise in which the core implosion of a supernova results in the creation of a black hole instead of a neutron star. Has this shown up in any of your calculations and, if so, what were the consequences?

Colgate: In the calculation of Dick White and myself the mass of the neutron star formed in the explosion was always significantly less than the currently accepted critical neutron star mass. As a consequence the Newtonian hydrodynamics used was justified. In the calculations of Arnett, on the other hand, more mass accumulated on the neutron star than would be stable in general relativity. The implication was that a black hole would have been formed had general relativistic hydrodynamics been used. Wilson has made general relativistic calculations that indeed show the formation of a black hole. To the extent that some mass is ejected there would be negligible observable difference for an outside observer. Of course the lack of a pulsar would be evident at some later date. 